bilden, die ich wegen der Schwierigkeit, sie in gröfserer Menge zu erhalten, nur unvollkommen untersuchen konnte. Ich hatte sie gleichwobl auf Grand einiger Annäherungsanalysen als das Phenylurethan der Methyl- und Aethylreihe

$$
\begin{aligned}
& \left.\mathrm{C}_{8} \mathrm{H}_{9} \mathrm{~N} \mathrm{O}_{2}=(\mathrm{C} \mathrm{O})^{\mathrm{\prime \prime}}\left(\mathrm{C}_{6} \mathrm{H}_{5}\right) \underset{\mathrm{CH} \mathrm{H}_{3}}{\mathrm{H} \mathrm{N}}\right\} \mathrm{O} \text { und }
\end{aligned}
$$

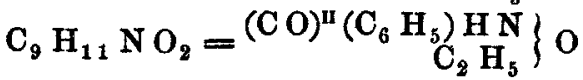

angesprochen.

Irgendwelche Zweifel über die Natur dieser Verbindungen, welche noch bätten geblieben sein können, sind durch die im vorhergehenden beschriebenen Versnche gelöst.

47. A. Keknlé: Witthoilungen aus dem chemischen Institnt der Universität Bonn.

1. Ueber eine neue Darstellungeweise des Cymols aus Campher und ein Oxyderivat des Cymols. Von Dr. R. Pott.

Um über die Constitution des Camphers neue Anhaltspunkte zu gewinnen, schien es von Interesse, die dem Campher entsprechenden Schwefelverbindungen darzustellen und zu untersuchen. Da nun die durch Einwirkung des Camphercblorids $\left(\mathrm{C}_{10} \mathrm{H}_{16} \mathrm{Cl}_{2}\right)$ auf Schwefelwasserstoffkalium und auf Schwefelkalium entstehenden Producte bis jetzt nicht rein erbalten werden konnten, so wurde die Einwirkung von Phosphorsulfid auf Campher versucht. Es zeigte sich, dafs so kein schwefelhaltiger Abkömmling des Camphers gebildet wird, dafs vielmehr, unter Entwicklung von Schwefelwasserstoff, reichliche Mengen von Cywol äberdestilliren. $\mathrm{Da}$ der so erhaltene Kohlenwasserstoff, nach vorhergegangener Behandlung mit Kalilange, fast vollständig zwischen $175-178^{\circ}$ überdestillirt, so scheint die angegebene Reaction eine zweckmäfsige Methode zur Darstellung des Cymols aus Campher abzugeben.

Die Identität des mittelst Phosphorsulfid dargestellten Cymols mit dem durch andere Reagentien aus Campher bereiteten Cymol wurde durch Bestimmung des Siedepunkts, durch Analyse und aufserdem durch das Aussehen and die Zusammensetzung des cymolsulfosauren Baryts festgestellt.

Wird cymolsulfosaures Kali (1 Th.) mit Kalihydrat (2 Th.) geschmolzen, das Product in Wasser gelöst, mit Schwefelsăure übersättigt und mit Wasserdampf destillirt, so geht ein gelbliches, etwas dickflüssiges Oel über, welches bei der Rectification fast vollständig bei $230^{\circ}$ überdestillirt. Die durch die Analyse gefundenen Werthe führen 
$\mathrm{zu}$ der Formel: $\mathrm{C}_{10} \mathrm{H}_{14} \mathrm{O}$, aber das so dargestellte Oxycymol weicht in seinen Eigenschaften von dem seither bekannten Thymol ab; es scheint mit demselben nur isomer, aber nicht identisch qu sein. Es ist flüssig, und konnte weder durch längeres Abkühlen noch dadurch zum Krystallisiren gebracht werden, dafs Krystalle des gewöhnlichen Thymols eingetragen wurden. Auch gegen concentrirte Schwefelsăure verhält es sich versehieden, es wird nur langsam und unvollständig in eine Sulfosäure übergeführt, deren wasserfreies Barytsalz in schönen Nadeln krystallisirt.

\section{Ueber die Einwirkung von Bromwasserstoff auf} Nitrobenzol. Von H. Baumbauer.

Jodwasserstoff reducirt bekanntlich die Nitrosubstitutionsproducte zu Amidoderivaten; aus Nitrobenzol entsteht bei etwa $104^{\circ}$ Anilin. Veranlafst durch Hrn. Dr. Glaser wurde statt des Jodwasserstoffs der weniger zersetzbare Bromwagserstoff angewandt, in der Hoffnung, dalsu so vielleicht das Product einer weniger weit gehenden Reduction, also etwa ein Nitrosokörpex, würde erhalten werden können.

Der Versuch lehrte, dafs Nitrobenzol von einer gesättigien wäfsrigen Lösung von Bromwasserstoff bei etwa $185-190^{\circ}$ zersetzt wird. Es entsteht dabei wesentlieb Dibromanilin und Tribromanilin, die leicht nach den bekannten Methoden getrennt werden können. Der Bromwasserstoff wirkt also, wenn auch erst bei höherer Temperatur, ganz in derselben Weise reducirend auf Nitrobenzol wie der Jodwasserstoff.

$$
\begin{aligned}
& \mathrm{C}_{6} \mathrm{H}_{5} \cdot \mathrm{NO}_{2}+6 \mathrm{HJ}=\mathrm{C}_{6} \mathrm{H}_{5} \cdot \mathrm{NH}_{2}+3 \mathrm{~J}_{2}+2 \mathrm{H}_{2} \mathrm{O} \\
& \mathrm{C}_{6} \mathrm{H}_{5} \cdot \mathrm{N} \mathrm{O}_{2}+6 \mathrm{HBr}=\mathrm{C}_{6} \mathrm{H}_{5} \cdot \mathrm{N} \mathrm{H}_{2}+3 \mathrm{Br}_{2}+2 \mathrm{H}_{2} \mathrm{O}
\end{aligned}
$$

$\mathrm{Da}$ aber das freiwerdende Brom ánf Anilinsalze substituirend einwirkt (was bei Jod nicht der Fall ist), so werden, statt des Anilins, Substitutionsproducte dieser Base erhalten. Wenn die Reaction ganz glatt verliefe und wenn alles Brom verbraucht würde, so würde nur Tribromanilin gebildet werden:

$$
\mathrm{C}_{6} \mathrm{H}_{5} \cdot \mathrm{NO}_{2}+6 \mathrm{HBr}=\mathrm{C}_{6} \mathrm{H}_{2} \mathrm{Br}_{3} \cdot \mathrm{NH}_{2}+3 \mathrm{BrH}+2 \mathrm{H}_{2} \mathrm{O}
$$
Auch die Chlorwasserstoffsäure wirkt bei $200-230^{\circ}$ auf Nitrobenzol ein; nach längerem Erhitzen setzen sic̈h farblose Octaeder und Würfel ab. Die Untersuchung der so erzeugten Producte ist indessen noch nicht beendigt.

HI. Ueber einige Reactionen der bromsalpetrigen Säure.

\section{Von L. de Koninck.}

Die Reactionen, über welche hier kurz berichtet werden soll, waurden auf Veranlassung von Hrn. Dr. Glaser ausgeführt; zunächst in der Absicht, zu versuchen, ob vermittelst der bromsalpetrigen Säure (N O Br) vielleicht Nitrosokörper würden erhalten werden können. 
Die bromsalpetrige Säure wurde, nach Landolt's Angaben, durch Sättigen von auf etwa $-10^{\circ}$ abgekühlten Brom mit Stickoxydgas dargestellt.

Die bromsalpetrige Säure könnte zunächst durch doppelten Austausch Nitrososubstitutionsproducte erzeugen; es ist indessen bis jetzt nicht gelungen, Reactionen der Art zu verwirklichen. Sie könnte sich dann weiter, ähnlich wie Bromwasserstoff, mit gewissen wasserstoffärmeren Substanzen vereinigen; die schon begonnenen Versuche lassen hoffen, dals so bromhaltige Nitrosoderivate erhalten werden können.

Eine glatt verlaufende Reaction der bromsalpetrigen Săure wurde bis jetzt nur bei einer Körpergruppe beobachtet, bei den Amidoderivaten. Dabei findet jedoch, wie za erwarten stand, eine ganz andere Art von Einwirkung statt; die bromsalpetrige Säure wirkt genau wie salpetrige Säure; aus den Amidoderivaten entstehen Diazokörper.

Lǘst man z. B. bromsalpetrige Saure, unter Abkühlen, auf in Wasser zertheiltes salpetersaures. Anilin einwirken, so wird ein Salz des Diazobenzols gebildet, aus welchem leicht krystallisirtes schwefelsaures Diazobenzol mit allen für diesen Körper charalteristischen Eigenschaften erhalten werden kann. Versetzt man eine alkobolische Lösung von Anilin mit in Alkohol gelöster bromsalpetriger Săute, so wird krystallisirbares Diazoamidobenzol erzengt, welches dann weiter in das durch sein salzsaures Salz so wohl charakterisirte Amidoazobenzol umgewandelt werden kann.

Diese Reactionen zeigen, dafs die bromsalpetrige Säure als das der salpetrigen Săure entsprechende Bromid angesehen werden kann, eine Annahme, mit der auch die übrigen Eigensehaften dieses Salpetrigsăurebromids übereinstimmen. Es liefert z. B. mit Kalilösung Bromkalium and salpetrigsaures Salz, und es greift Quecksilberoxyd schon in der Kälte an, indem, neben Quecksilberbromid, Salpetrigsäureanhydrid gebildet wird.

\section{Correspondenzen.}

48. Ch. Friedel, ans Paris am 20. März.

Die Sitzung der Akademie vom 15. laufenden Monats bot nur wenige chemische Abhandlungen. Hr. Chancel überreichte durch Hrn. Cahours eine Notiz über den Gährungspropylalkobol. Die Einzelheiten dieser Arbeit sind in der Sitzung nicht mitgetheilt worden, sondern nur das allgemeine Resnitat, welches mit dem von Hrn. Fittig und Hrn. $J_{s i d}$ r Pierre angegebenen, vollständig übereinstimmt, dals nämlich dieser Alkohol das wahre Homologe des Aethylalkohols ist. 